\title{
DYNAMIC SOCIALITY MINORITY GAME
}

\author{
Franco Cicirelli, Angelo Furfaro, Libero Nigro, Francesco Pupo \\ Laboratorio di Ingegneria del Software \\ Dipartimento di Elettronica Informatica e Sistemistica \\ Università della Calabria \\ 87036 Rende (CS) - Italy \\ Email: \{f.cicirelli,a.furfaro\}@deis.unical.it, \{1.nigro,f.pupo\}@unical.it
}

\section{KEYWORDS}

Minority game, social ability, multi-agent systems, simulation

\begin{abstract}
The minority game (MG) is a simple yet effective binarydecision model which is well suited to study the collective emerging behaviour in a population of agents with bounded and inductive rationality when they have to compete, through adaptation, for scarce resources. The original formulation of the MG was inspired by the W.B. Arthur's El Farol Bar problem in which a fixed number of people have to independently decide each week whether to go to a bar having a limited capacity. A decision is only affected by information on the number of visitors who attended the bar in the past weeks. In its basic version, the MG does not contemplate communication among players and it supposes that information about the past game outcomes is publicly available. This paper proposes the Dynamic Sociality Minority Game (DSMG), an original variant of the classic MG where (i) information about the outcome of the previously played game step is assumed to be known only by the agents that really attended the bar the previous week and (ii) a dynamically established acquaintance relationship is introduced to propagate such information among non attendant players. Particular game settings are identified which make DSMG able to exhibits a better coordination level among players with respect to standard MG. Behavioral properties of the DSMG are thoroughly analyzed through an agent-based simulation of a simple road-traffic model.
\end{abstract}

\section{INTRODUCTION}

The Minority Game (MG) (Challet and Zhang, 1997) is an inductive game born as a mathematical formulation of the El Farol Bar attendance problem (Arthur, 1994). The problem refers to a scenario in which a fixed number of people have to decide about making use of a shared resource represented by a bar. Since the space in the bar is limited (finite resources), the sojourn is considered enjoyable only if the number of attendances remains under a specified threshold. Basic formulation of MG considers $N$ (supposed odd) players that make a choice be- tween two options at each turn, e.g. to attend the bar or to stay home. Winners are those that belong to the minority side, i.e. that chosen by at most $(n-1) / 2$ players. Each player is initially fed with a fixed and randomly chosen set of strategies that it may use to calculate its next choice on the basis only of the past outcomes of the game. The game generalizes the study of how many individuals, competing in a resource constrained environment, may reach a collective solution to a problem under adaptation of each one's expectation about the future without resorting to cooperation strategies.

MG proved to be effective in many application fields like economics, biology and social science. In (Lustosa and Cajueiro, 2010) the game is applied in a scenario tied to market of goods being auctioned. In (Cicirelli et al., 2007) MG is exploited for developing protocols controlling the coverage level in large wireless sensor networks. Applications related to predator/prey models and traffic scenarios can be found respectively in (Cicirelli et al., 2011) and (Bazzan et al., 2000)(Chmura and Pitz, 2006). The game has been the subject of extensive study (Challet et al., 2005) and the original model has been modified in various ways (Sysi-Aho, 2005). Adaptive minority game models, in which agents try to improve their performances by modifying their strategies through genetic algorithm based on crossover mechanisms, are discussed in (Sysi-Aho et al., 2004). The concept of agent personality is used in (Bazzan et al., 2000) where players are equipped with a selected combination of game strategies trying to model certain types of human behaviour. Social relationships among players are considered in (Remondino and Cappellini, 2005). Here it is introduced the concept of Local Minority Game where each player owns a local view of the game and it will win or lose a game step on the basis of the decisions taken by the players belonging to its acquaintances. Each game step is divided in two phases: in the first phase each player undertakes a non-committed choice which is made available to the acquaintances, in the second phase each player uses such shared information to commit its previously made choice or definitively change it. In this approach the established social relations cannot change dynamically.

The Constrained Information Minority Game (CIMG) (Lustosa and Cajueiro, 2010) shares with the work proposed in this paper the basic idea that the only players 
who surely know the outcome of the last turn of the game are those who actually attended the bar. This is a realistic hypothesis because it considers that, in a population, information about a happening propagates in a different way. In the CIMG, though, it is assumed that not attendant players may become aware of the last game outcome only on the basis of a fixed probability.

In this paper, the Dynamic Sociality Minority Game (DSMG) is proposed. DSMG assumes that (i) information about the outcome of the previously played game step is only known by players that really attended the bar and (ii) a dynamically established acquaintance relationship is available to propagate such information among non attendant players. Here it is argued that the capability of exploiting dynamic sociality behavior is an important issue for modeling scenarios arising in the daily life in a more realistic way. For instance, supposing that a player can move over a territory, it becomes possible to take into account situations where acquaintances depend on the specific position owned by a player during a game step. Moreover the number of acquaintances may change over the time and may be related also to the ability of a player to establish (or to keep alive) social relations with other people in its nearness.

Behavioral properties of DSMG are studied by using a modelling example based on a simple road traffic scenario. A single road connecting two places, e.g. a city and a resort, is considered. People have to decide if to go on holyday or returning home avoiding traffic. DSMG analysis is carried out through agent based simulation (Macal and North, 2006)(Wickenberg and Davidsson, 2003). The Theatre agency (Cicirelli et al., 2009) is used for implementing the game. Simulation results show that game settings exist which make DSMG able to exhibits a better coordination level among players with respect to standard MG. Moreover, when the number of acquaintances reduces, the game results not organized around the socially optimal point, for instance this means that the road in the adopted example may be underutilized on the average, and arbitrage opportunities may arise (Lustosa and Cajueiro, 2010). The rest of the paper is structured as follows. First the definition of the DSMG is provided, then its behavioural properties are discussed through the road traffic model. Finally, conclusions are given with an outlook of future work.

\section{DYMANIC SOCIALITY MINORITY GAME}

In the basic formulation of MG (Challet and Zhang, 1997), $N$ (supposed odd) players make a binary choice attempting to be in the minority side. Each player is initially fed with a randomly chosen set $S$ of strategies that it uses to calculate its next choice on the basis only of the past $M$ outcomes of the game. Since there are only two possible outcomes, $M$ is also the number of bits needed to store the history of the game. The number of possible histories is of course $P=2^{M}$, strategies are numerable and their number is $2^{P}$. Players rank their own strategies based on their respective capability to predict the winner side. Every player associates each strategy with a virtual score which is incremented every time the strategy, if applied, would have predicted the minority side. A penalty is instead assigned to bad behaving strategies. At each game step, a player uses the first ranked strategy. When there is a tie among possible strategies, the player chooses randomly among them. Standard MG assumes that information about last game step is publicly available. As a consequence the same history exists for all players.

At each game step, DSMG groups players in three categories named participant (PA), informed (IN) and noninformed (NI). PA are those players that really attended the bar and directly know the game outcome. IN represents players that although did not go to the bar, they indirectly know the game outcome through their social network. NI denotes non-attendant players which remain unaware of the last game outcome. NI players are not able to update their strategies nor their history. As a consequence, players in the DSMG may accumulate a different history and may have a different view on the whole game status.

DSMG can be formally defined as in the following. Let $O=\{-1,+1\}$ be the set of possible outcomes of the game, $P L$ be the set of players and $I$ be a subset of natural numbers corresponding to the game steps. Let $h: P L \times I \rightarrow O^{M}$ be the function modelling the history of a player, i.e. $h(p, i)$ returns the last $M$ outcomes of the game of player $p$, preceding a given game step $i ; h(p, 0)$ is randomly set for each player. Let $S=\left\{S_{1}, \ldots, S_{n}\right\}$ be the set of all allowed strategies and $S_{j}: O^{M} \rightarrow O$ be a strategy function which guesses the next winner side by looking at the game history. Let $s t r: P L \times I \rightarrow N$ be the function which returns the index of the strategy used by player $p$ at the game step $i$. The outcome of a player $p$ at game step $i$ is given by $S_{s t r(p, i)}^{h(p, i)}$. Once all players have defined their choice at step $i$, the sum of these choices defines the outcome of that step: $A(i)=$ $\sum_{p \in P L} S_{s t r(p, i)}^{h(p, i)}$. Let $A c q: P L \times I \rightarrow 2^{P L}$ be a function determining the set of acquaintances of player $p$ at the game step $i$, and Cat : $P L \times I \rightarrow\{P A, I N, N I\}$ be the function which determines the category of player $p$ at game step $i$ :

$$
\operatorname{Cat}(p, i)= \begin{cases}P A & \text { if } S_{s t r(p, i)}^{h(p, i)}=+1 \\ I N & \text { if } S_{s t r(p, i)}^{h(p, i)}=-1 \wedge \\ & \exists p^{\prime} \in \operatorname{Acq}(p, i): \operatorname{Cat}\left(p^{\prime}, i\right)=P A \\ N I & \text { otherwise }\end{cases}
$$

Let $V_{S_{j}}: P L \times I \rightarrow \mathbb{Z}$, where $\mathbb{Z}$ is the set of integer, be the virtual score assigned by player $p$ to strategy $S_{j}$ at game step $i . V_{S_{j}}(p, 0)=0$ for each strategy and for each player. Virtual scores are updated according to the following rule:

$V_{S_{j}}(p, i+1)=\left\{\begin{array}{lr}V_{S_{j}}(p, i) & \text { if } C a t(p, i)=N I \\ V_{S_{j}}(p, i)-S_{s t r(p, i)}^{h(p, i)} A(i) & \text { otherwise }\end{array}\right.$ 
Similarly, the history function of a player $p$ at game step $i$ is not updated in the case $\operatorname{Cat}(p, i)=N I$.

\section{DSMG BEHAVIOR}

DSMG behavior was studied through an example based on a simple traffic model. The model assumes that a road connects two places, namely a city and a resort. An odd number of players are randomly split between the two places. At every game step, a player who is in the city(resort) has to decide whether to make a trip toward the resort(city) or to avoid traveling. A player having +1 as outcome is supposed to make the trip. A player having -1 as outcome does not make use of the road. The road has a limited traffic capacity and, as consequence, the enjoyable choice is that done by the minority of players. A player which does not travel may ask to other players in the same place, i.e. its acquaintances, about traffic news. Due to the departures and the arrivals, the identity and the number of players in a place changes over the time. Analysis of DSMG is carried out by using agent based simulation. The Theatre agency (Cicirelli et al., 2009), whose strength comes from the exploitation of a light-weight thread-less actor (agent) framework, is used for implementing the game. Actors are used both to model the application business logic and players behavior. In addition to $A(i)$, the observable measures of the game are: (i) the average of the game outcomes $M_{A}=\frac{1}{T} \sum_{i=1}^{T} A(i)$, where $T$ denotes the played game steps, (ii) the per-capita fluctuation of the game outcomes $\sigma^{2} / N$, where $N$ denotes the number of players and $\sigma^{2}=\frac{1}{T} \sum_{i=1}^{T}\left(A(i)-M_{A}\right)^{2}$. Average and per-capita fluctuation are also evaluated separately for the number of participant and non-informed players. A fixed number of $N=101$ players and $T=5000$ game steps are considered in all the game settings. Different configurations of the game are achieved by varying (i) the number of acquaintances that a player may contact at each game step, (ii) the history size $M$ and (iii) the number of strategies assigned to players. In particular $M \in\{2,4,6,8\},|A c q| \in\{1,11,31,41,51\}$, \#strategies $=\{2,6,10,14\}$. Two different schemas were considered in order to assign strategies to players. In the first schema, strategies are randomly chosen among those admissible. In the second schema, tied only to $M=2$, some artificial (i.e. wayward) players are also considered, i.e. players for which strategies are purposely selected.

\section{Players having a randomly chosen set of strategies}

Fig. 1 shows the observed measures of DSMG in the case the history of the game has a length of 8 bits. As one can see, as the number of acquaintances increases, both the average of game outcomes and its per-capita fluctuation tend to be the same as for standard MG regardless the number of strategies assigned to each players (Fig. 1(a) and Fig. 1(b) ). This is also confirmed in Figures from $1(\mathrm{e})$ to $1(\mathrm{~h})$ where the number of non-informed players tends to be zero as the number of acquaintances reaches 11. By cross-referencing data from Fig. 1(c) to 1(f) it emerges that, except for the case of 1 acquaintance, players' population can be roughly partitioned in only participant and informed. This means that the established social network is able to propagate information to all the non-attendant players. DSMG with $M=8$ appears to perform worse than or equal to standard MG in that it exhibits a less coordination level among players as the number of acquaintances decreases. Simulation results show that the trend inverts as soon as a shorter memory is exploited. In particular, in Fig. 2 are portrayed DSMG observables in the case $M=2$. The per-capita fluctuation reaches a minimum by considering 11 acquaintances and 2 strategies for each player (see Figures from 2(b) to 2(d)). Also for $M=2$, the trade-off for changing game behavior remains 11 acquaintances (see Fig. 2(e) and Fig. 2(f)). As a consequence of increasing the number of acquaintances above such a value, the number of non-informed players goes to zero.

A more detailed view of the per-capita fluctuation of game outcomes is portrayed in Fig. 3. Fluctuation is studied by considering different values of the control parameter $z=\frac{2^{M}}{N}$ (Liaw et al., 2007). By using respectively 2 (Fig. 3(a) ) and 6 (Fig. 3(b)) strategies for each player, two different scenarios are considered by varying the size of the acquaintance relationship. Dashed lines refer to the standard MG. With the exception of the setting of 2 strategies - 1 acquaintance, DSMG results in a better coordination among players. In particular a good level of coordination is achieved in the low- $z$ region (i.e. $z \ll 1$ ). In (Liaw et al., 2007) it is argued that the high fluctuation of standard MG in the low- $z$ region is due to the fact that too many players switch simultaneously. Therefore, a winning prediction (minority) turns into a losing one (majority). Liaw et al. confirmed experimentally that the average number of switching players is large in the low- $z$ region and that by artificially limiting the number of switching players the fluctuation of game outcomes quickly reduces to a small value. DSMG is naturally able to limit the number of switching players. In fact, a non-attending non-informed player "freezes" its choice also in the next game step (this is because both strategy scores and history remain unchanged).

Even if the per-capita fluctuation of game outcomes heavily depends on the game settings, the time series of the number of players that decided to stay at the resort (or equivalently in the city) remains instead almost unchanged. It was observed that the average value of the players located at the resort varies in the interval $[50.35,50.67]$ in a way that is not related to fluctuation of game outcomes. Per-capita fluctuation of players at the resort ranges from 0.2 to 0.54 . Fig. 4 reports two of such time series.

\section{Artificial players}

The exploitation of artificial players in the DSMG can be justified by two specific reasons. As a first concern, 


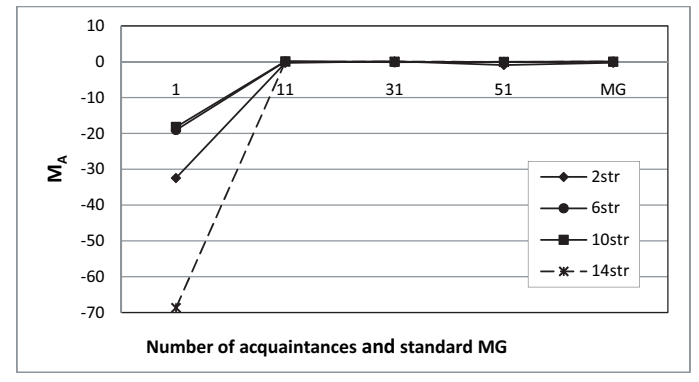

(a) Average of game outcomes

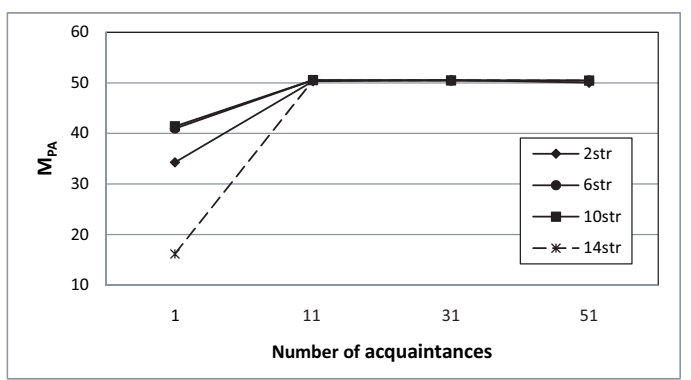

(c) Average number of participant players

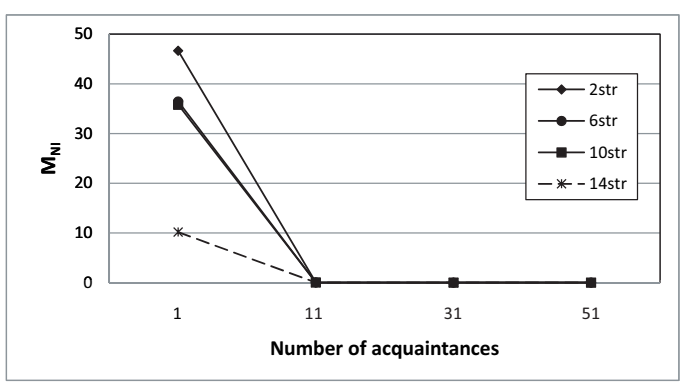

(e) Average number of non-informed players

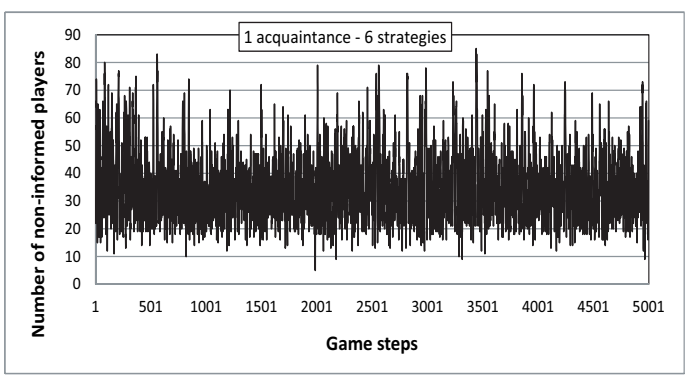

(g) Non-informed players vs. game steps

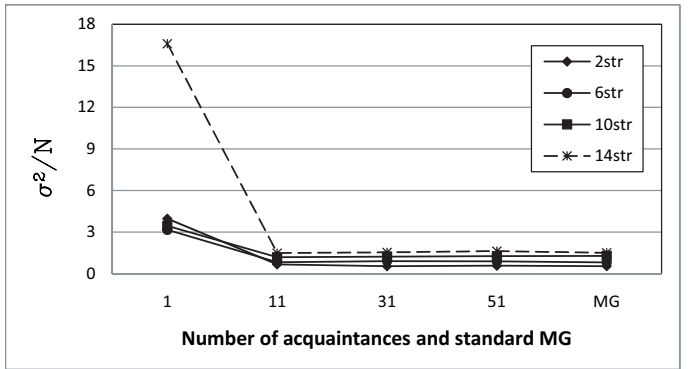

(b) Per-capita fluctuation of game outcomes

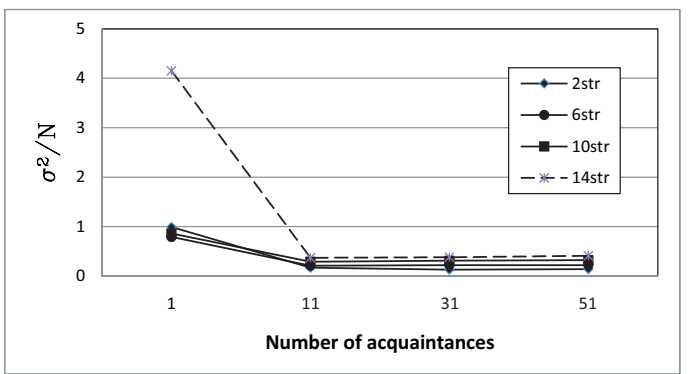

(d) Per-capita fluctuation of number of participant players

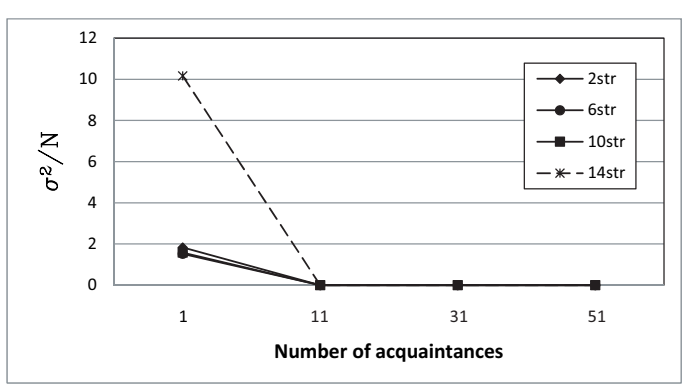

(f) Per-capita fluctuation of number of non-informed players

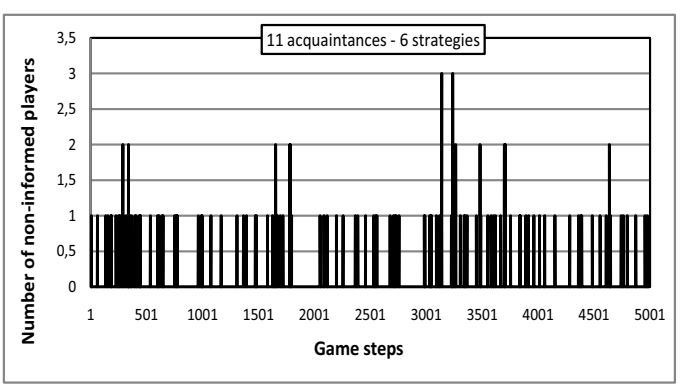

(h) Non-informed players vs. game steps

Figure 1: Observables of DSMG with $M=8$ 


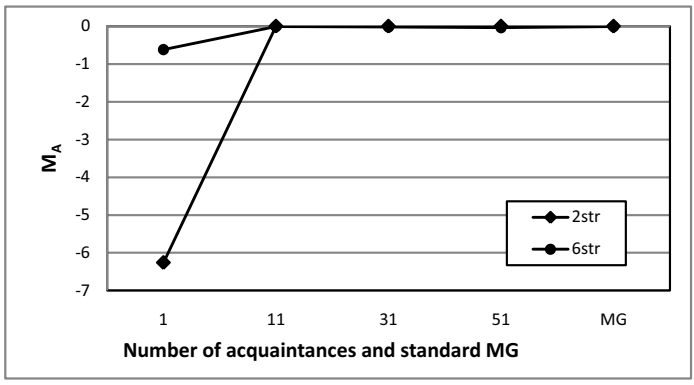

(a) Average of game outcomes

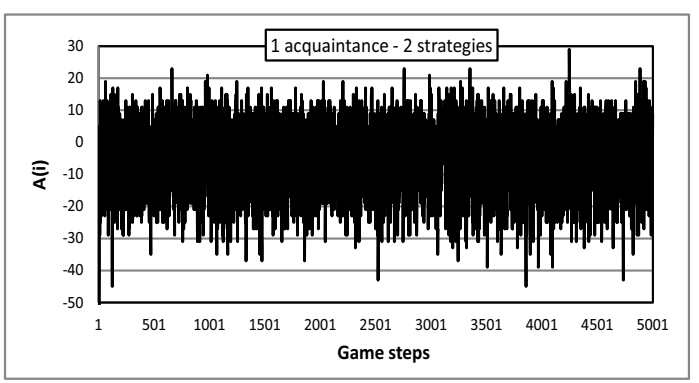

(c) Outcome of the game vs. game steps

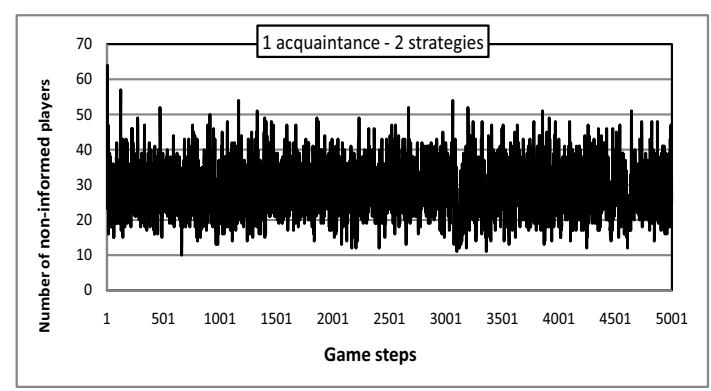

(e) Non-informed players vs. game steps

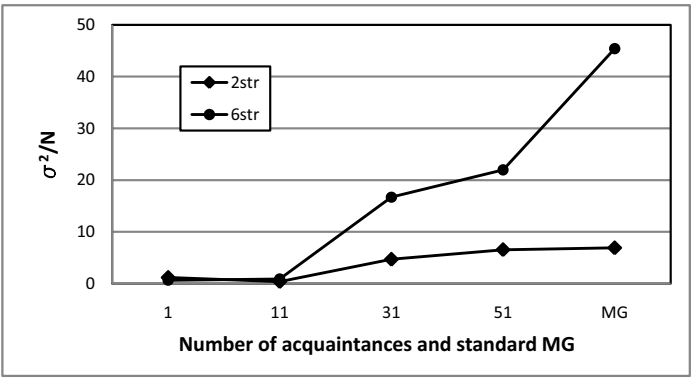

(b) Per-capita fluctuation of game outcomes

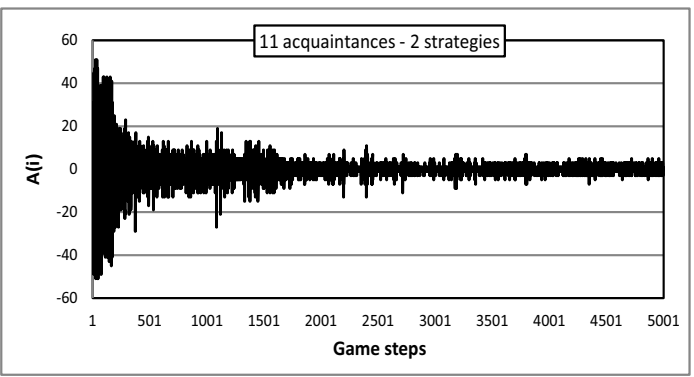

(d) Outcome of the game vs. game steps

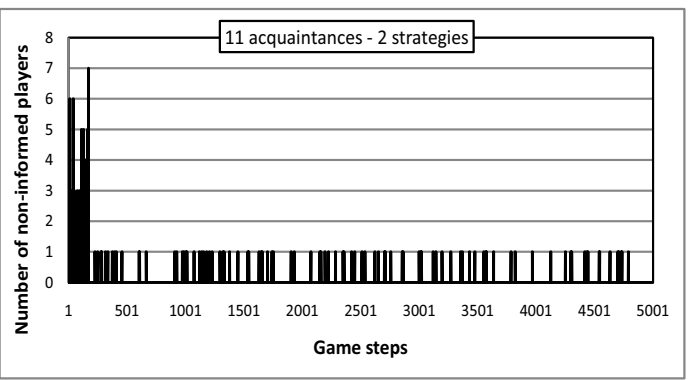

(f) Non-informed players vs. game steps

Figure 2: Observables of DSMG with $M=2$

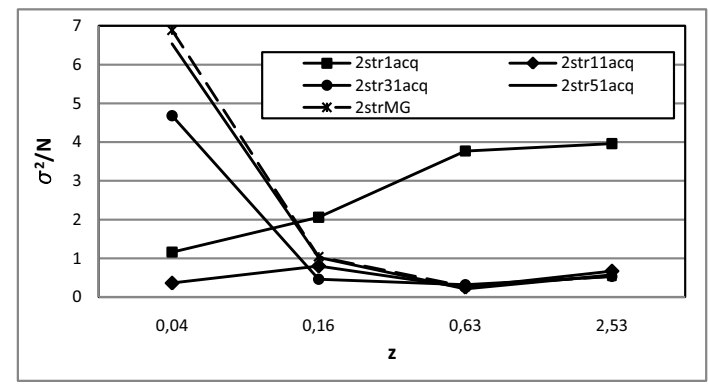

(a) 2 strategies and a variable number of acquaintances

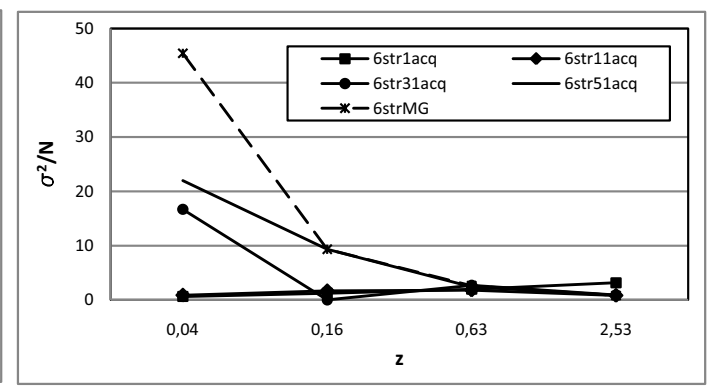

(b) 6 strategies and a variable number of acquaintances

Figure 3: Per-capita fluctuation of game outcomes vs. $z$ 


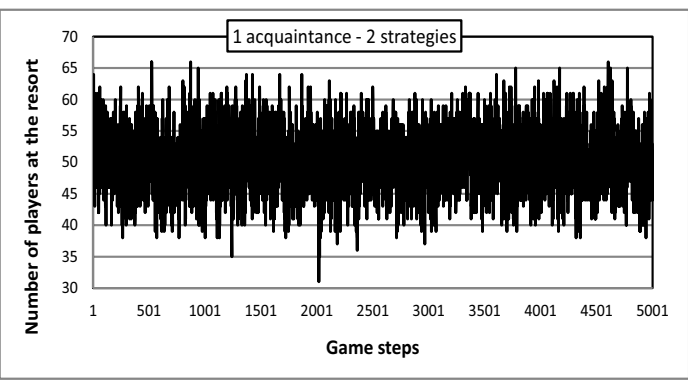

(a)

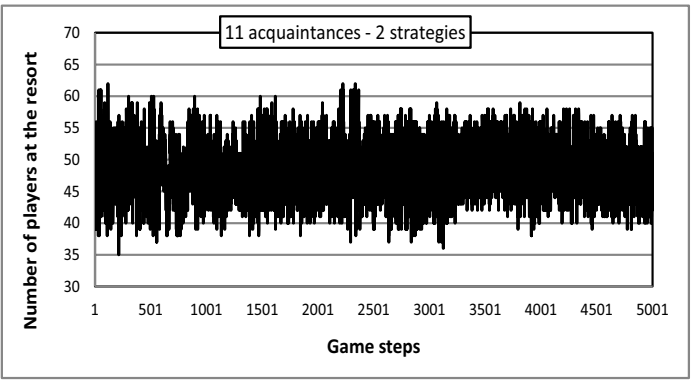

(b)

Figure 4: Number of players at the resort vs. game steps $(M=2)$

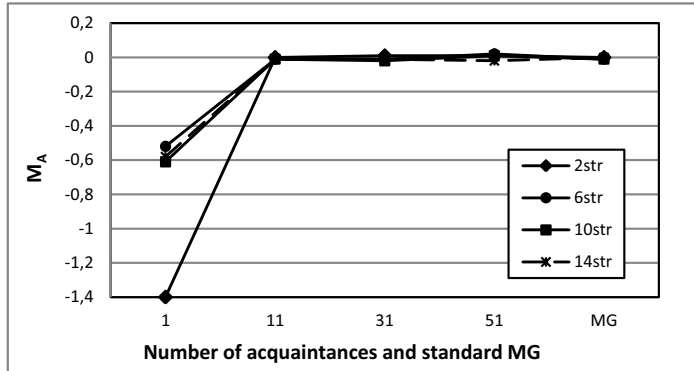

(a) Average of game outcomes

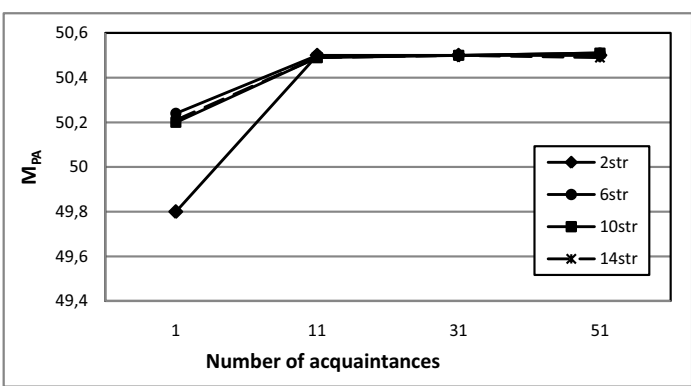

(c) Average number of participant players

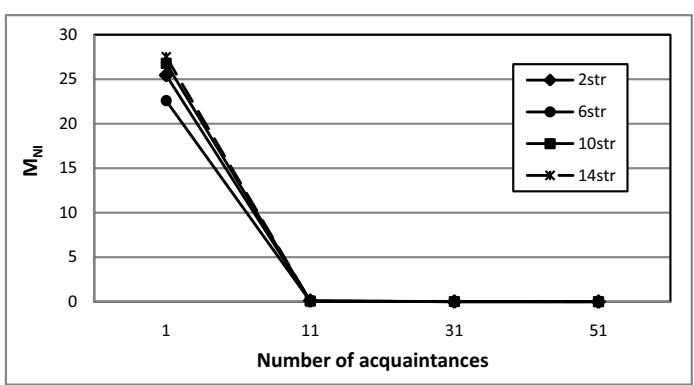

(e) Average number of non-informed players

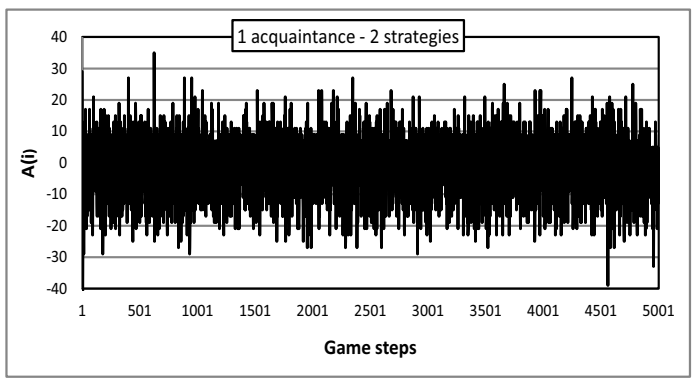

(g) Outcome of the game vs. game steps

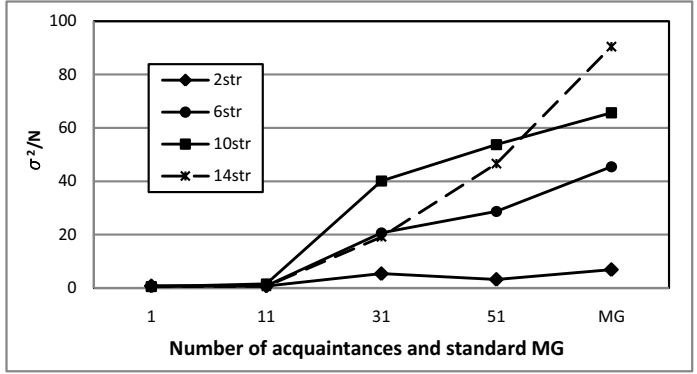

(b) Per-capita fluctuation of game outcomes

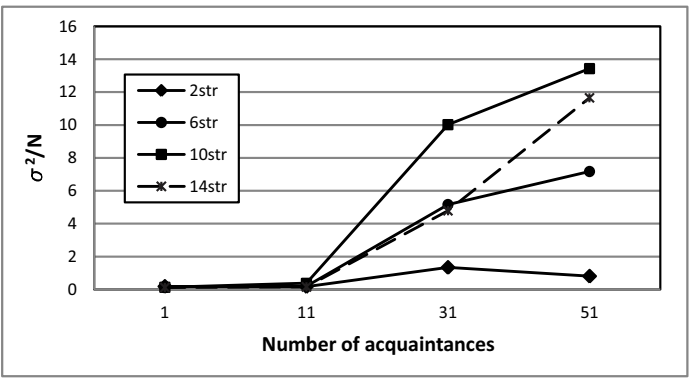

(d) Per-capita fluctuation of number of participant players

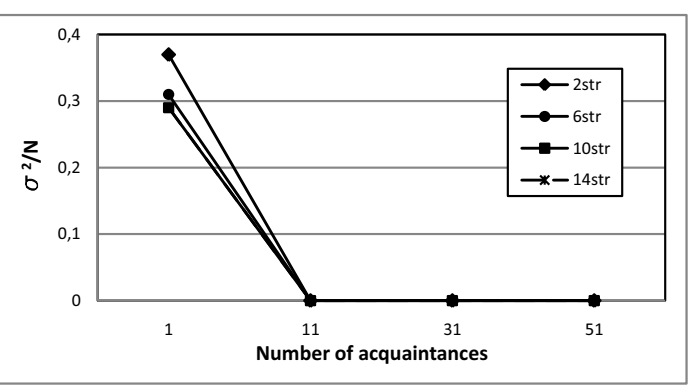

(f) Per-capita fluctuation of number of non-informed players

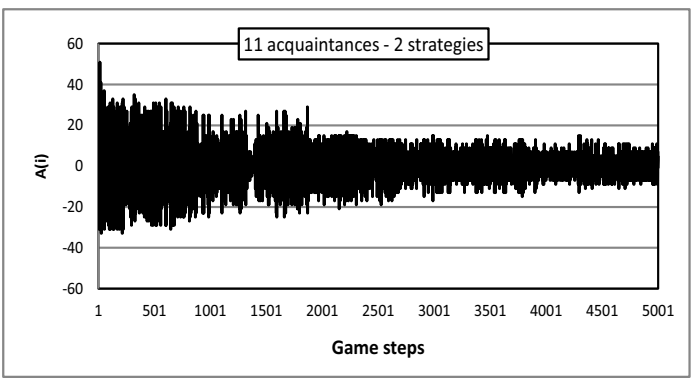

(h) Outcome of the game vs. game steps

Figure 5: Observables of DSMG with $M=2$ and $10 \%$ of "wayward" players 
from Figures 2(a) and 3(a) it clearly emerges that, in the case the number of acquaintances is less than 11, the game does not appear organized around the socially optimal point. As a consequence, arbitrage opportunities may arise (Lustosa and Cajueiro, 2010). Arbitrage refers to the possibility of a risk-free profit at zero cost. This means that, since in the proposed traffic scenario the road is underutilized, some wayward players would think to augment their "wealth" by simply making use of the road at each game step. The second reason is concerned with the need to avoid impasse situations during the DSMG evolution. Let suppose, for instance, that during a game step the number of participant players is zero. From this point on, all players will remain in the non-informed category and, as a consequence, the game will hang. Simulation experiments confirm that such a situation arises e.g. in the case of $M=2$ and a number of strategies set to 10 or 14 . This is why Figures 2(a) and 2(b) only refer to 2 and 6 strategies.

In order to cope with the two above described problems, some players were equipped with strategies enforcing a single-minded behavior always leading to making use of the road independently of the game history. Fig. 5 refers to a scenario where $M=2$ and the $10 \%$ of population is constituted by "artificial" players. Fig. 5(a) shows that the average of game outcomes, with 1 acquaintance, is closer to zero than that of Fig. 2(a). As the number of acquaintances increases, the average tends to zero as in the normal version of the game. In this new scenario the game never hangs.

\section{CONCLUSIONS}

This paper proposes the Dynamic Sociality Minority Game (DSMG), an original variant of the classic Minority Game (MG) where (i) information about the outcome of the previously played game step is assumed to be known only to players that actually attended "the bar the previous week" and (ii) a dynamically established acquaintance relationship is introduced to propagate, on demand, such information to non attendant players. Observable measures of the game were studied through an example based on a simple road traffic model. DSMG behavior was analyzed and compared with that of standard MG. Specific game settings were identified which make DSMG able to exhibit a better coordination level among players with respect to standard MG. Moreover, by reducing the number of acquaintances, the game does not appear organized around the socially optimal point and, therefore, arbitrage opportunities may arise. On-going and future work is geared at:

- investigating further game measures in the case the size of the acquaintance relationship reduces

- extending the game definition by taking into account local minorities and player spatial coordinates influencing the acquaintance relationships
- adopting more realistic scenarios so as to evaluate players' social abilities

- experimenting with DSMG in the distributed simulation of predator/prey models (Cicirelli et al., 2011).

\section{REFERENCES}

Arthur, W. (1994). Inductive reasoning and bounded rationality. The American Economic Review, 84(2):406-411.

Bazzan, A. L. C., Bordini, R. H., Andrioti, G. K., Vicari, R. M., and Wahle, J. (2000). Wayward agents in a commuting scenario (Personalities in the minority game). In Proc. Fourth Int MultiAgent Systems Conf, pages 55-62.

Challet, D., Marsili, M., and Zhang, Y. (2005). Minority games. Oxford University Press.

Challet, D. and Zhang, Y. C. (1997). Emergence of cooperation and organization in an evolutionary game. Physica A: Statistical and Theoretical Physics, 246(3-4):407-418.

Chmura, T. and Pitz, T. (2006). Successful strategies in repeated minority games. Physica A: Statistical Mechanics and its Applications, 363(2):477-480.

Cicirelli, F., Furfaro, A., Giordano, A., and Nigro, L. (2011). Performance of a multi-agent system over a multi-core cluster managed by terracotta. In Proc. of Symposium On Theory of Modeling and Simulation - DEVS Integrative M\&S Symposium (TMS/DEVS'11), Boston, MA, USA.

Cicirelli, F., Furfaro, A., and Nigro, L. (2007). Exploiting agents for modelling and simulation of coverage control protocols in large sensor networks. Journal of Systems and Software, 80(11):1817-1832.

Cicirelli, F., Furfaro, A., and Nigro, L. (2009). An agent infrastructure over HLA for distributed simulation of reconfigurable systems and its application to UAV coordination. SIMULATION, 85(1):17-32.

Liaw, S., Hung, C., and Liu, C. (2007). Three phases of the minority game. Physica A: Statistical Mechanics and its Applications, 374(1):359-368.

Lustosa, B. and Cajueiro, D. (2010). Constrained information minority game: How was the night at El Farol? Physica A: Statistical Mechanics and its Applications, 389(6):12301238.

Macal, C. M. and North, M. J. (2006). Tutorial on agent-based modeling and simulation part 2: How to model with agents. In Proc. the Winter Simulation Conf., pages 73-83.

Remondino, M. and Cappellini, A. (2005). Minority game with communication of statements and memory analysis: a multiagent based model. International Journal of Simulation, $6(5): 42-53$.

Sysi-Aho, M. (2005). A Game Perspective to Complex Adaptive Systems. PhD thesis, Department of Electrical and Communications Engineering, Helsinki University of Technology, Espoo, Finland.

Sysi-Aho, M., Chakraborti, A., and Kaski, K. (2004). Searching for good strategies in adaptive minority games. Physical Review E, 69(3):036125.

Wickenberg, T. and Davidsson, P. (2003). On multi agent based simulation of software development processes. In Proc. of the 3rd Int. Conf. on Multi-agent-based simulation II, pages 171-180, Berlin, Heidelberg. 